\title{
FACTORING FOURIER TRANSFORMS WITH ZEROS IN A STRIP
}

\author{
D. G. DICKSON
}

(Communicated by Paul S. Muhly)

\begin{abstract}
Fourier transform of an infinitely differentiable function of compact support on $\mathbf{R}$ if, and only if, $f$ is entire and of exponential type with $|f(x)|=O\left(|x|^{-N}\right)$ for each $N>0$ as $|x| \rightarrow \infty$ for real $x$. In some sense, such an $f$ has its zeros close to the real axis and has positive density of zeros $F$ with $n(r)=D r+o(r)$. It is shown here that if the zeros of $f$ are in a strip parallel to the real axis and if $n(r)=D r+O(1)$, then $f$ is the product of two such transforms with zero densities $D / 2$ and indicators one-half of the indicator of $f$. There is a factorable $f$ in $\widehat{\mathscr{D}}(\mathbf{R})$ with zeros on a line and not satisfying the stricter density condition. Analogous results hold for transforms of distributions of compact support on $\mathbf{R}$. The study was motivated by the outstanding problem of Ehrenpreis that asks if $\mathscr{D}(\mathbf{R}) * \mathscr{D}(\mathbf{R})=\mathscr{D}(\mathbf{R})$.
\end{abstract}

\section{INTRODUCTION}

Let $\mathscr{F}=\widehat{\mathscr{D}}(\mathbf{R})$ and $\mathscr{G}=\widehat{\mathscr{E}}^{\prime}(\mathbf{R})$ denote, respectively, the algebras of all Fourier transforms of infinitely differentiable functions of compact support on the real line $\mathbf{R}$ and of distributions of compact support on $\mathbf{R}$. By the PaleyWiener-Schwartz theorem, $\mathscr{F}$ and $\mathscr{G}$ are the sets of entire functions of exponential type, with those in $\mathscr{F}$ satisfying $|f(x)|=o\left(|x|^{-N}\right)$ as $|x| \rightarrow \infty$ for each positive integer $N$ when $x$ is real, and those in $\mathscr{G}$ satisfying $|f(x)|=o\left(|x|^{N}\right)$ for some positive integer $N$ as $|x| \rightarrow \infty$. Multiplication of functions in $\mathscr{F}$ and $\mathscr{G}$ corresponds to convolution in $\mathscr{D}(\mathbf{R})$ and $\mathscr{E}^{\prime}(\mathbf{R})$.

In [6] Ehrenpreis asked if $\mathscr{D}(\mathbf{R}) * \mathscr{D}(\mathbf{R}) \rightarrow \mathscr{D}(\mathbf{R})$ is a surjective map; equivalently, is each $f$ in $\mathscr{F}$ the product of functions in $\mathscr{F}$ ? A negative response to the same question has been established in $\mathscr{D}\left(\mathbf{R}^{n}\right)$ for $n \geq 2$ in [2], [7], and [8], while the question remains unanswered in $\mathscr{D}(\mathbf{R})$. Although the corresponding question in $\mathscr{G}$ is trivially true using a polynomial factor, a more significant question in $\mathscr{G}$ is whether factors can be found that halve the density of zeros and the indicator function of the product. The factorability of functions in $F$ that are real on the real axis was established in [3] and was easily extended under the same condition to $\mathscr{G}$ in [4]. In [5] it was shown that factorability

Received by the editors July 14, 1988.

1980 Mathematics Subject Classification (1985 Revision). Primary 30D15, 30C15; Secondary 44A35, 46F 10 . 
was possible in larger classes of functions and in $\mathscr{F}$ and $\mathscr{G}$ when density and monotonicity conditions were imposed on the zeros of $f$. Here it is shown that a slightly stronger density condition will yield factorability when the zeros of $f$ lie in a strip parallel to the real axis.

The main tool used here is the factorability in $\mathscr{F}$ and $\mathscr{G}$ of functions with only real zeros $[3,4,8]$. Unfortunately, we are able to exhibit a function in $\mathscr{F}$ that is factorable (since it has only real zeros) and does not satisfy the stronger density condition.

\section{Preliminaries}

Let $f$ be in $\mathscr{G}$. Suppose $[a, b]$ is the interval of support of the inverse Fourier transform of $f,\left\{z_{n}=r_{n} \exp \left(i \theta_{n}\right)\right\}$ is the set of non-zero zeros of $f$ ordered by non-decreasing modulus, and $n(r)$ is the number of zeros of modulus at most $r$. Then $\lim _{r \rightarrow \infty} n(r) / r=D>0$, and the series $\sum \cos \theta_{n} / r_{n}$ and $\sum\left|\sin \theta_{n}\right| / r_{n}$ converge. The fact that the second series converges is the sense in which the zeros are said to be close to the real axis. Actually, the zeros have equal right and left-hand plane densities. The indicator function of $f$ is

$$
h_{f}(\theta)=\varlimsup_{r \rightarrow \infty} \frac{\log \left|f\left(r e^{i \theta}\right)\right|}{r}=\max \{a \sin \theta, b \sin \theta\} .
$$

$D=(b-a) / \pi$, and $f$ has the product representation

$$
f(z)=e^{-i(a+b) z / 2+d} z^{m} \prod_{n=1}^{\infty}\left(1-\frac{z}{z_{n}}\right) .
$$

For our factorization considerations there is no loss of generality in assuming that $d=m=a+b=0$. Then

$$
f(z)=\prod_{n=1}^{\infty}\left(1-\frac{z}{z_{n}}\right)
$$

while $D=2 b / \pi$ and $h_{f}(\theta)=b|\sin \theta|$. Note that products by polynomials or division by polynomial factors of functions in $\mathscr{F}$ and $\mathscr{G}$ are still in $\mathscr{F}$ or $\mathscr{G}$, respectively. $\mathscr{F}$ and $\mathscr{G}$ are also translation invariant since $f(z)$ is the transform of $u(t)$ if, and only if, $f(z+\tau)$ is the transform of $\exp (-i \tau t) u(t)$. Some of these facts and appropriate references may be found in the book [1] by Boas.

\section{FACTORIZATION}

The content of this investigation is contained in the following theorem.

Theorem. $f$ is in $\mathscr{F}$ (resp. $\mathscr{G}$ ) with zeros $\left\{z_{n}\right\}$ contained in a strip parallel to the real axis and $n(r)=D r+O(1)$. Then $f=f_{1} f_{2}$ for two functions $f_{1}$, and $f_{2}$ in $\mathscr{F}$ (resp. $\mathscr{G}$ ); each factor has a zero set of density $D / 2$ and an indicator function one-half of the indicator of $f$.

Proof. The proof will be written for $f$ in $\mathscr{F}$ with the very few adjustments for $f$ in $\mathscr{G}$ indicated when they arise. The broad approach will be the following: 
Beginning with $f$ in $\mathscr{F}$, auxiliary functions $F$ and $G$ with real zeros will be constructed. $F$ will be in $\mathscr{F}$ since it will be dominated in modulus on $\mathbf{R}$ by $f . G$ will be in $\mathscr{F}$ since it will be comparable to $F$ on $\mathbf{R}$ by our density assumption. Using our earlier theorem, $G$ will be factored in $\mathscr{F}$. By a vertical translation, those factors will be transformed to functions in $\mathscr{F}$ that will dominate corresponding factors of $f$ in modulus on $\mathbf{R}$, ensuring their inclusion in $\mathscr{F}$.

Assume that $f$ has the form (1). In view of the previously indicated invariances of $\mathscr{F}$, we may assume that the zeros of $f$ are in the strip $0 \leq \operatorname{Im}(z) \leq \beta$ and that $\left|\operatorname{Re}\left(z_{n}\right)\right|>2 \beta$ for each zero $z_{n}$. Two sequences $\left\{t_{n}\right\}$ and $\left\{\tau_{n}\right\}$ of real numbers will be constructed from $\left\{z_{n}\right\}$ to serve as zeros of $F$ and $G$. With $z=r \exp (i \theta)$, the plane level curves $C$ of $r /|\cos \theta|$ are circles tangent to the imaginary axis at the origin; let $C_{n}$ be the level curve through $z_{n}$. Since $\left|\operatorname{Re}\left(z_{n}\right)\right|>2 \beta, z_{n}$ lies on the upper outside (away from the imaginary axis) quarter of $C_{n}$. Let $t_{n}=r_{n} / \cos \theta_{n}$ so that $t_{n}$ is the non-zero point of intersection of $C_{n}$ and the real axis. Then let $\tau_{n}=\left(t_{n} \pm\left(t_{n}^{2}-4 \beta^{2}\right)^{1 / 2}\right) / 2$ using the positive root when $t_{n}>0$ and the negative root when $t_{n}<0$. Then $\tau_{n}+i \beta$ is the upper outside point of intersection of $C_{n}$ and the line $\operatorname{Im}(z)=\beta$. We will show that $\mathscr{F}$ contains

$$
F(z)=\prod_{n=1}^{\infty}\left(1-\frac{z}{t_{n}}\right) \text { and } G(z)=\prod_{n=1}^{\infty}\left(1-\frac{z}{\tau_{n}}\right) .
$$

For $z=r \exp (i \theta)$ on $C_{n}, r / \cos \theta=t_{n}$, and for all real $x$,

$$
1-\frac{x}{z}=1-\frac{x}{t_{n}}+i \frac{x \sin \theta}{r} \text {. }
$$

Since the level curves of $r /|\sin \theta|$ are circles tangent to the real axis at the origin, it is clear that $|1-x / z|$ increases as $z$ traces the upper half of $C_{n}$ in the direction toward the imaginary axis. As a result,

$$
\left|1-\frac{x}{t_{n}}\right| \leq\left|1-\frac{x}{z_{n}}\right| \leq\left|1-\frac{x}{\tau_{n}+i \beta}\right| \text {. }
$$

Since $f$ is in $\mathscr{F}, \sum 1 / t_{n}$ converges and $\left\{t_{n}\right\}$ has the density $D$ of $\left\{z_{n}\right\}$. Lindelöf's theorem implies that $F$ is entire and of exponential type. By (3), $|F(x)| \leq|f(x)|$ for all real $x$, so $F$ is in $\mathscr{F}$.

While it is easy to show that $G$ is entire and of exponential type, it is less easy to compare $|G(x)|$ with $|F(x)|$. To accomplish the first of these, we use the easily established facts that $\left|t_{n}-\tau_{n}\right|<\beta$ and $t_{n} \tau_{n}>t_{n}^{2} / 2$, so that

$$
\left|\frac{1}{t_{n}}-\frac{1}{\tau_{n}}\right| \leq \frac{\left|\tau_{n}-t_{n}\right|}{t_{n} \tau_{n}} \leq \frac{2 \beta}{t_{n}^{2}} .
$$

Since $F$ is of exponential type, $\sum 1 / t_{n}^{2}$ converges. Using the Cauchy criterion, the convergence of $\sum 1 / t_{n}$ and the last inequality, it is easy to see that $\sum 1 / \tau_{n}$ 
also converges. Since $\left\{\tau_{n}\right\}$ also has density $D, G$ is entire and of exponential type.

We now proceed to show that $|G(x)|$ is appropriately bounded on $\mathbf{R}$ so that $G$ will be in $\mathscr{F}$ (or $\mathscr{G}$ ). We will compare values of $A(t)=|1-x / t|$ when $t$ is $t_{n}$ and $t$ is $\tau_{n}$ or $\tau_{n+1}$. Since $A(t)$ has different monotonicity intervals determined by $t=0$ and $t=x$, direct comparisons of $A\left(\tau_{n}\right)$ and $A\left(t_{n}\right)$ are not always productive; also, it is often desirable to compare $A\left(\tau_{n+1}\right)$ and $A\left(t_{n}\right)$ but $t_{n}$ and $\tau_{n+1}$ are not consistently ordered in the monotonicity intervals unless $t_{n}$ and $t_{n+1}$ are separated. The desired separation can result by using subsequences. The density assumption insures that this separation is possible using a finite number of subsequences, which in turn results in the required bounds for $G$ on $\mathbf{R}$ and insures that $G$ is in $\mathscr{F}$.

The fact that $n(r)=D r+O(1)$ implies that there is an integer $N>0$ such that each square of side $\beta$ contains $z_{n}$ for at most $N$ values of $n$. We will now split the sequence $\left\{z_{n}\right\}$ into at most $2 N$ subsequences.

Let $j$ be an integer with $|j| \geq 2$. Let

$$
R_{j}=\{z=x+i y ; j \beta \leq x<(j+1) \beta, 0 \leq y \leq \beta\} \quad \text { for } j \geq 2
$$

and

$$
R_{j}=\{z=x+i y ;(j-1) \beta<x \leq j \beta, 0 \leq y \leq \beta\} \quad \text { for } j \leq-2 .
$$

Then the $R_{j}$ are square tiles (lacking their outside edges) whose union covers $\left\{z_{n}\right\}$. For indices $j$ going from 1 to $\infty$, let $T_{1}=\cup R_{2 j+1}, T_{2}=\cup R_{2 j}$, $T_{-1}=\cup R_{-2 j-1}$, and $T_{-2}=\cup R_{-2 j}$. Consecutive squares in each $T_{j}$ are separated by squares of side $\beta$. Since $f$ has equal positive densities of zero in the right and left half planes, at least one of $T_{1}$ and $T_{2}$ and at least one of $T_{-1}$ and $T_{-2}$ contain infinitely many zeros. We will suppose each of the four $T_{j}$ contains infinitely many zeros with obvious modifications possible otherwise. We will also suppose that (a) the maximum number of $z_{n}$ in a squares of $T_{j}$ is $N_{j} \leq N$, (b) the first square of $T_{j}$ contains $N_{j}$ zeros, and (c) infinitely many squares of $T_{j}$ contain $N_{j}$ zeros. These assumptions may be justified by the invariances of $\mathscr{F}$ indicated earlier.

The set of zeros $z_{n}$ in each $T_{j}$ may be partitioned into $N_{j}$ subsequences $S_{j p}, p=1,2, \ldots, N_{j}$, in such a way that no two $z_{n}$ in $S_{j p}$ are in the same $R_{k}$. Let

$$
S_{j p}=\left\{z_{j p q}\right\}_{q=1}^{\infty} \quad \text { with } z_{j p 1} \text { in } R_{j}
$$

and for $z_{j p q}$ in $R_{k}, z_{j p q+1}$ is in some $R_{k^{\prime}}$ with $\left|k^{\prime}\right| \geq|k|+2$. Then the real parts of consecutively indexed zeros in $S_{j p}$ are separated by at least $\beta$.

The following separation assertion is easily proved since the rectangles are separated:

$$
\begin{gathered}
z_{\lambda} \in R_{k}, \quad z_{\mu} \in R_{k^{\prime}}, \quad k k^{\prime}>0, \quad\left|k^{\prime}\right| \geq|k|+2 \\
\Rightarrow\left|t_{\mu}-t_{\lambda}\right|>2 b / 3 \text { and }\left|t_{\lambda}\right|<\left|\tau_{\mu}\right| .
\end{gathered}
$$


To establish bounds on $|G(x)|$ we will consider only the case $x>0$ since a symmetric argument can be used for $x<0$. We will compare terms $F_{\lambda}=$ $\left|1-x / t_{\lambda}\right|$ and $G_{\mu}=\left|1-x / \tau_{\mu}\right|$.

For $j=-1,-2$ and $p=1,2, \ldots, N_{j}$, in each of $S_{-1 p}$ and $S_{-2 p}$,

$$
\tau_{j p q+1}<t_{j p q}<0 \text { and } G_{j p q+1}<F_{j p q}
$$

by (4) and the fact that $A(t)$ is increasing for $t<0$. Hence for each $r>0$,

$$
\prod_{-r<\tau j p q} G_{j p q}<G_{j p 1} \prod_{-r<\tau_{j p q}} F_{j p q} .
$$

For $j=1,2$ and $p=1,2, \ldots, N_{j}$, choose $k=k(j, p)$ so that $\tau_{j p k} \leq x<$ $\tau_{j p k+1}$. In each of $S_{1 p}$ and $S_{2 p}, t_{j p q}<\tau_{j p q+1}$ by (4). Since $A(t)$ decreases in $(0, x]$ and increases in $(x, \infty)$,

$$
\begin{array}{ll}
G_{j p q}<F_{j p q-1} & \text { for } q=1, \ldots, k(j, p), \text { and } \\
G_{j p q}<F_{j p q} & \text { for } q \geq k(j, p)+1 .
\end{array}
$$

Combining these, for $r>x+4 \beta$,

$$
F_{j p k(j, p)} \prod_{\tau j p q<r} G_{j p q} \leq G_{j p 1} \prod_{\tau_{j p q}<r} F_{j p q} .
$$

Combining (5) and (6) by taking products over $p$ and over $j$,

$$
\left[\prod_{j=1}^{2} \prod_{p=1}^{N_{j}} F_{j p k(j, p)}\right] \prod_{\left|\tau_{\lambda}\right|<r} G_{\lambda} \leq\left[\prod_{j=-2}^{2} \prod_{p=1}^{N_{j}} G_{j p 1}\right] \prod_{\left|\tau_{\lambda}\right|<r} F_{\lambda} .
$$

The first term of the left member is the modulus of a product of $N_{1}+N_{2}=Q$ factors of the form $1-x / t_{\lambda}$ where the $\lambda$ 's depend on $x$; denote the product by $P_{x}(x)$. The first term of the right member is a product of $\sum_{-2}^{2} N_{j}=P$ terms of the form $\left|1-x / \tau_{j p 1}\right|$ each of which is bounded by $\beta^{-1}(1+\beta)(1+|x|)$ since $\left|\tau_{\lambda}\right| \geq\left|\operatorname{Re}\left(z_{\lambda}\right)\right|>2 \beta$; denote the product bound by $B(1+|x|)^{P}$. Then, letting $r \rightarrow \infty$, (7) takes the form

$$
|G(x)| \leq B(1+|x|)^{P}\left|F(x) / p_{x}(x)\right|
$$

where $p_{x}(z)$ is a polynomial factor of $F(z)$ of degree $Q$. By a corollary to a simple theorem in [3, p. 88], if $F$ is in $\mathscr{F}$ and $Q$ is a positive integer, then there are $\gamma_{N}$ and $R_{N}$ with the property that

$$
|F(x) / p(x)| \leq \gamma_{N}(1+|x|)^{-N} \quad \text { for all } x>R_{N}
$$

and all polynomial factors $p(z)=\Pi\left(1-z / z_{\lambda}\right)$ of $F(z)$ of degree $Q$. (A similar statement for some $N>0$ and $|x|^{N}$ holds when $f$ is in $\mathscr{G}$.) It then follows from (8) that $G$ is in $\mathscr{F}$.

Since $G$ is real on the real axis, it follows from [3] that $G=G_{1} G_{2}$ where the $G_{j}$ are in $\mathscr{F}$ and have zero densities $D / 2$. Let $\sigma_{j}$ denote the set of zeros of 
$G_{j}$. In order to simplify notation, limits as $r \rightarrow \infty$ of sums or products over $\tau_{\lambda}$ in $\sigma_{j}$ with $\left|\tau_{\lambda}\right|<r$ will be denoted by $\sum_{j}$ and $\Pi_{j}$. Then, for $j=1,2$,

$$
G_{j}(z)=\prod_{j}\left(1-\frac{z}{\tau_{\lambda}}\right) .
$$

By translation, $H_{j}(z-i \beta)$ is in $\mathscr{F}$ for each $j$, and

$$
H_{j}(z)=\prod_{j}\left(1-\frac{z-i \beta}{\tau_{\lambda}}\right)=G_{j}(-i \beta) \prod_{j}\left(1-\frac{z}{\tau_{\lambda}+i \beta}\right) .
$$

Since $\sum_{j} 1 / \tau_{\lambda}$ and $\sum_{j} 1 / \tau_{\lambda}^{2}$ converge, it follows from the Cauchy criterion and

$$
\left|\frac{1}{z_{\lambda}}-\frac{1}{\tau_{\lambda}}\right| \leq \frac{\left|z_{\lambda}-\tau_{\lambda}\right|}{\left|z_{\lambda}\right|\left|\tau_{\lambda}\right|} \leq \frac{\beta}{\tau_{\lambda}^{2}}
$$

that $\sum_{j} 1 / z_{\lambda}$ converges. Then also

$$
\sum_{\left|z_{\lambda}<\right| r, \tau_{\lambda} \in \sigma_{j}} \frac{1}{z_{\lambda}}
$$

converges. Hence we may define, for $j=1,2$,

$$
f_{j}(z)=\lim _{r \rightarrow \infty} \prod_{\left|z_{\lambda}\right|<r, \tau_{\lambda} \in \sigma_{j}}\left(1-\frac{z}{z_{\lambda}}\right) .
$$

Each $f_{j}$ is then entire and of exponential type. With $x$ real, (3) together with (9) and (10) imply that $\left|f_{j}(x)\right| \leq\left|G_{j}(-i \beta)\right|^{-1}\left|H_{j}(x)\right|$. Since the $H_{j}$ are in $\mathscr{F}$, it follows that the $f_{j}$ are in $\mathscr{F}$. Then $f=f_{1} f_{2}$ is a factorization in $\mathscr{F}$. Clearly the $H_{j}$ and $f_{j}$ have the zero density $D / 2$ as do the $G_{j}$. Since there is no exponential term in the representation of the $f_{j}$, the center of the indicator diagrams of the $f_{j}$ is the origin. Since the density of $f_{j}$ is $D / 2=b \pi$ and is the length of the indicator diagram divided by $\pi$, the length of that diagram is $b$ so the indicator function for each $f_{j}$ is $b|\sin \theta| / 2$. This completes the proof of the theorem.

\section{AN EXAMPLE}

To show that not all factorable functions with zeros in a strip satisfy the density restriction $n(r)=D(r)+O(1)$, we construct a function in $\mathscr{F}$ with real zeros and zeros of arbitrarily high order. In [3] a function $f$ in $\mathscr{F}$ was constructed from $z^{-1} \sin \pi z$ by removing the zeros at $\pm(n !)^{2} 2^{n}$. We will now construct an entire function $g$ of exponential type that (a) has only real zeros, (b) has zeros of arbitrarily high order, and (c) is bounded on the real line. Then $h=g f$ will be factorable in $\mathscr{F}$ and have zeros of arbitrarily high multiplicity.

To this end, we use the facts that when $z=x+i y,\left|x^{-1} \sin x\right| \leq 1$ and $\left|z^{-1} \sin z\right| \leq e^{|y|} \leq e^{|z|}$. Then,

$$
\left|\frac{\sin \left(\pi z / 2^{n}\right)}{\pi z / 2^{n}}\right| \leq e^{\pi|z| / 2^{n}} .
$$


Let

$$
g(z)=\prod_{n=1}^{\infty} \frac{\sin \left(\pi z / 2^{n}\right)}{\pi z / 2^{n}} .
$$

$|g(z)| \leq \exp \left(\pi z \sum 1 / 2^{n}\right)=\exp (\pi z)$, and $|g(x)| \leq 1$. Hence $g$ is of exponential type and bounded on the real axis. $g$ has exactly $k$ zeros at $z= \pm 2^{k}$.

\section{REFERENCES}

1. R. P. Boas, Entire functions, Academic Press, New York, 1954.

2. J. P. Demailly, Construction d'hypersurfaces irréductibles avec lieu singulier donné dans $\mathbf{C}^{n}$, Ann. Inst. Fourier 30 (1980), No. 3, 219-236.

3. D. G. Dickson, Factoring rapidly decreasing entire functions, Bull. Sci. Math. (2) 110 (1986), 335-345.

4. _ Factoring Fourier transforms, Proceedings of the International Conference on Complex Analysis and its Applications, Varna, 1987, to appear.

5. __ Factoring functions of exponential type with restrictions on a line, Bull. Sci. Math. (2) 112 (1988), 77-100.

6. L. Ehrenpreis, Solutions of some problems of division IV, Amer. J. Math. 82 (1960), 522-588.

7. P. Malliavin and J. Dixmier, Factorisations de fonctions et de vecteurs indéfiniment différentiables, Bull. Sci. Math. (2) 102 (1978) 305-330.

8. L. A. Rubel, W. A. Squires and B. A. Taylor, Irreducibility of certain entire functions with applications to harmonic analysis, Annals of Math. (2) 108 (1978), 553-567.

Department of Mathematics, University of Michigan, Ann Arbor, Michigan 48109 